\title{
Corrosion Products Present in Wood Recovered from a Historic Shipwreck.
}

\author{
M. W. Pendleton,* G. Fox,** E. A. Ellis,* and B. B. Pendleton***
}

* Microscopy and Imaging Center, Texas A\&M University, Biological Sciences Building Room Number 119, Mail Stop 2257, College Station, TX. 77843-2257

** Museum of Anthropology, Department of Anthropology, California State University, Chico, Chico, CA. 95929-0400

*** Department of Agricultural Sciences, P.O. Box 60998, West Texas A\&M University, Canyon, TX. 79016-0001.

The whaling vessel Parker was wrecked in 1842 on the Kure Atoll reef, part of the Northwestern Hawaiian Islands Marine National Monument. In 2002 the wreck was discovered on the seaward side of the reef and within the lagoon of Kure Atoll. Among the scatter of artifacts recovered in 2005 was a copper ship's bell and the wood and iron yoke which supported it on the Parker. Scanning electron microscopy (SEM) was utilized to examine the wood samples of the Parker bell yoke. Samples were prepared for SEM by fixing with $2.5 \%$ (vol./vol.) acrolein in 0.1 M HEPES buffer ( $\mathrm{pH}$ 7.4), washed in buffer and dehydrated in methanol into hexamethyldisilazane. The dry samples were vapor coated with rhuthenium tetroxide [1] prior to SEM imaging using a JEOL JSM $6400 \mathrm{SEM}(30 \mathrm{KeV}$ accelerating voltage and $15 \mathrm{~mm}$ working distance). Crystals were observed on the outer surfaces of the yoke wood samples. During crystal observation, an Energy Dispersive Spectrometry (EDS) detector (Princeton Gamma-Tech (Bruker AXS, Inc., Madison, WI), with a takeoff angle of 31.0 degrees, count rates of $300-500 \mathrm{cps}$ for $100 \mathrm{~s}$ and an estimated probe current of $5.0 \mathrm{nA}$ ), was used to determine that these crystals contained iron, probably as a corrosion product. Figure 1 shows the EDS plot of the crystals shown in Fig. 2A with strong iron, carbon, and silicon peaks. The secondary SEM micrograph (Fig. 2A) demonstrates the crystal structure present over much of the surface of the yoke wood. Figure 2B is an EDS map which shows the location of X-ray signals of iron located in areas which correspond to the same areas of the secondary SEM micrograph (Fig. 2A). The x-ray signals of iron in Figure 2B appear to be evenly distributed over the entire surface of the crystals.

Although corrosion products associated with wood can have casts which mirror the original wood structure [2], the surface of the yoke from the Parker did not appear to have the original wood structure preserved. SEM micrographs of the crystals on the surface of the yoke wood were produced using both secondary and backscatter SEM detectors. The same area of the yoke wood is shown in Figures 3A (secondary detector) and 3B (backscatter detector). While the secondary micrograph (Fig. 3A) shows the surface details of the crystals, the backscatter micrograph (Fig. 3B) demonstrates the crystal structures at some depth so that the intersecting crystal growth patterns can be easily observed. The intersecting crystal growth pattern effectively obscured much of the detail of the yoke wood.

References:

[1] E. Ellis and M. Pendleton, Micros. Today 15 No. 3 (2007) 44.

[2] C. Keepax, J. Archeol. Sci. 2 No. 2 (1975) 145

[3] Artifacts described in this abstract are being analyzed at the Heritage Resources Conservation Laboratory, Department of Anthropology, California State University, Chico. The analysis is funded by a federal contract through the National Oceanic and Atmospheric Administration. 


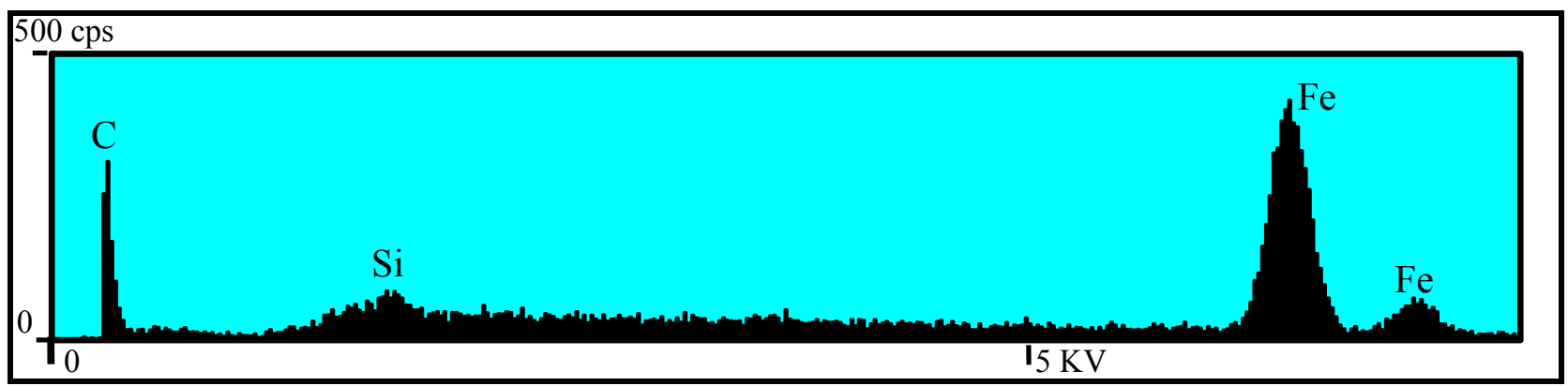

Fig. 1. EDS plot of shipwreck wood area shown in Fig. 2A (30 KeV, x-ray counting time $100 \mathrm{~s})$.
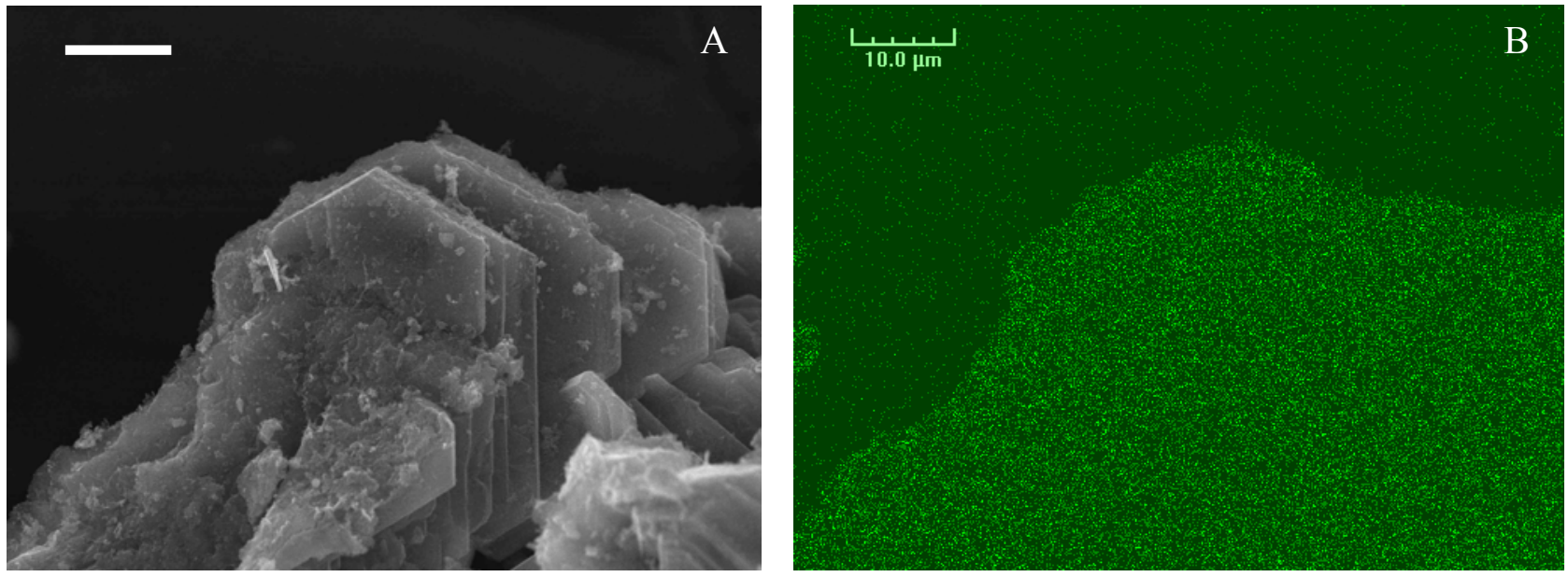

Fig. 2. Crystal structure of corrosion product. A. Secondary SEM micrograph of crystals on surface. Scale $=10 \mu \mathrm{m}$. B. EDS FeK $\alpha$ map of iron in area shown in Fig. 2A. Scale $=10 \mu \mathrm{m}$.
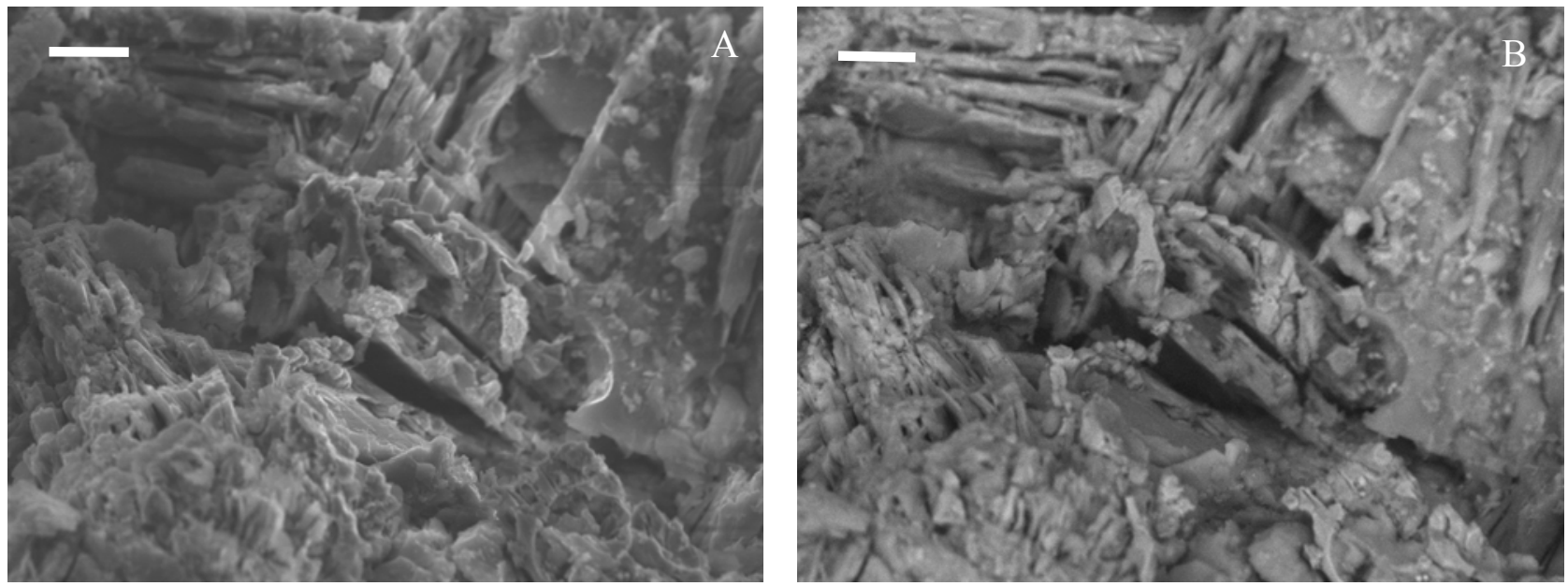

Fig. 3. SEM Micrographs of crystal growth on wood surface. A. Secondary SEM micrograph of crystals containing iron on wood surface. Scale bar $=10 \mu \mathrm{m}$. B. Backscatter SEM micrograph of crystals containing iron on wood surface of area shown in Fig. 3A. Scale bar $=10 \mu \mathrm{m}$. 